\title{
L'usine hydraulique d'accumulation journalière par pompage de Revin
}

\author{
par J. Ponsy \\ Chef du Service Etudes "Pompages" \\ E.D.F., R.E.H. Nord, Plobsheim
}

\section{Caractéristiques générales de l'aménagement:}

- Puissance maximale........ 800 MVA.

- Hauteur de chute moyenne... $230 \mathrm{~m}$.

- Capacité des bassins...... 7 millions de $\mathrm{m}^{3}$.

Description des principaux ouvrages:

- Bassin supérieur.

- Bassin inférieur.

- Adductions.

- Usine souterraine.

Examen de quelques problèmes particuliers :

- Marnages fréquents et rapides des retenues.

- Blindages des adductions souterraines.

- Prises d'eau.

L'aménagement de Revin constitue la première réalisation par E.D.F. d'une usine hydraulique d'accumulation journalière par pompage.

\section{1. - Le site}

L'aménagement est implanté dans le massif des Ardennes à proximité de la frontière franco-belge, et à $180 \mathrm{~km}$ environ de Paris. Ce massif se présente sous forme d'un vaste plateau, couvert par une abondante végétation forestière, et entaillé par des vallées encaissées.

Sous une couverture variable mais en général assez faible de limons et éboulis, le socle rocheux est constitué de schistes et quartzites de formation très ancienne (cambrien supérieur). Superficiellement délité et altéré, le rocher devient très rapidement sain en profondeur; en grand, il est homogène et imperméable; sa qualité est suffisante pour permettre l'ouverture de grandes excavations souterraines dans de bonnes conditions, moyennant quelques précautions d'exécution.
Le site de Revin est favorable à l'installation d'une station de pompage:

- par ses caractéristiques topographiques qui offrent la possibilité d'équiper une puissance suffisamment importante sous une hauteur de chute de $230 \mathrm{~m}$ entre deux bassins voisins;

- par ses caractéristiques géologiques qui permettent une implantation souterraine des adductions et de l'usine.

En plus de ses qualités intrinsèques, le site jouit d'une situation géographique privilégiée à proximité des grands centres de consommation d'énergie de la région parisienne et des régions industrielles du nord de la France dont les moyens de production sont essentiellement thermiques.

\section{2. - Caractéristiques générales (figure 1)}

L'aménagement utilise la dénivellation qui existe entre le plateau qui culmine à la cote 400 du N.G.F. et la vallée de la Faux, petit affluent rive gauche de la Meuse qui coule aux environs de la cote 145 N.G.F.

- Le bassin supérieur est un réservoir entièrement artificiel, du type lac collinaire, aménagé sur le plateau des Marquisades.

- Le bassin inférieur est créé par une digue d'une quarantaine de mètres de hauteur qui barre le lit de la Faux.

Les volumes utiles des deux bassins sont de $7 \mathrm{Mm}^{3}$ et la dénivellation brute entre les deux plans d'eau varie avec le marnage entre 220 et $246 \mathrm{~m}$.

La distance horizontale entre les deux bassins est de lordre de $1100 \mathrm{~m}$.

- Les adductions qui les relient sont entièrement souterraines. Elles transitent des débits maximaux de $300 \mathrm{~m}^{3} / \mathrm{s}$ en pompage et $400 \mathrm{~m}^{3} / \mathrm{s}$ en turbinage.

- L'usine souterraine, implantée à proximité du bassin inférieur, est équipée de quatre groupes «turbine-pompe » réversibles, d'une puissance unitaire de $200 \mathrm{MVA}$. Cet équipement correspond à un cycle journalier d'exploitation 


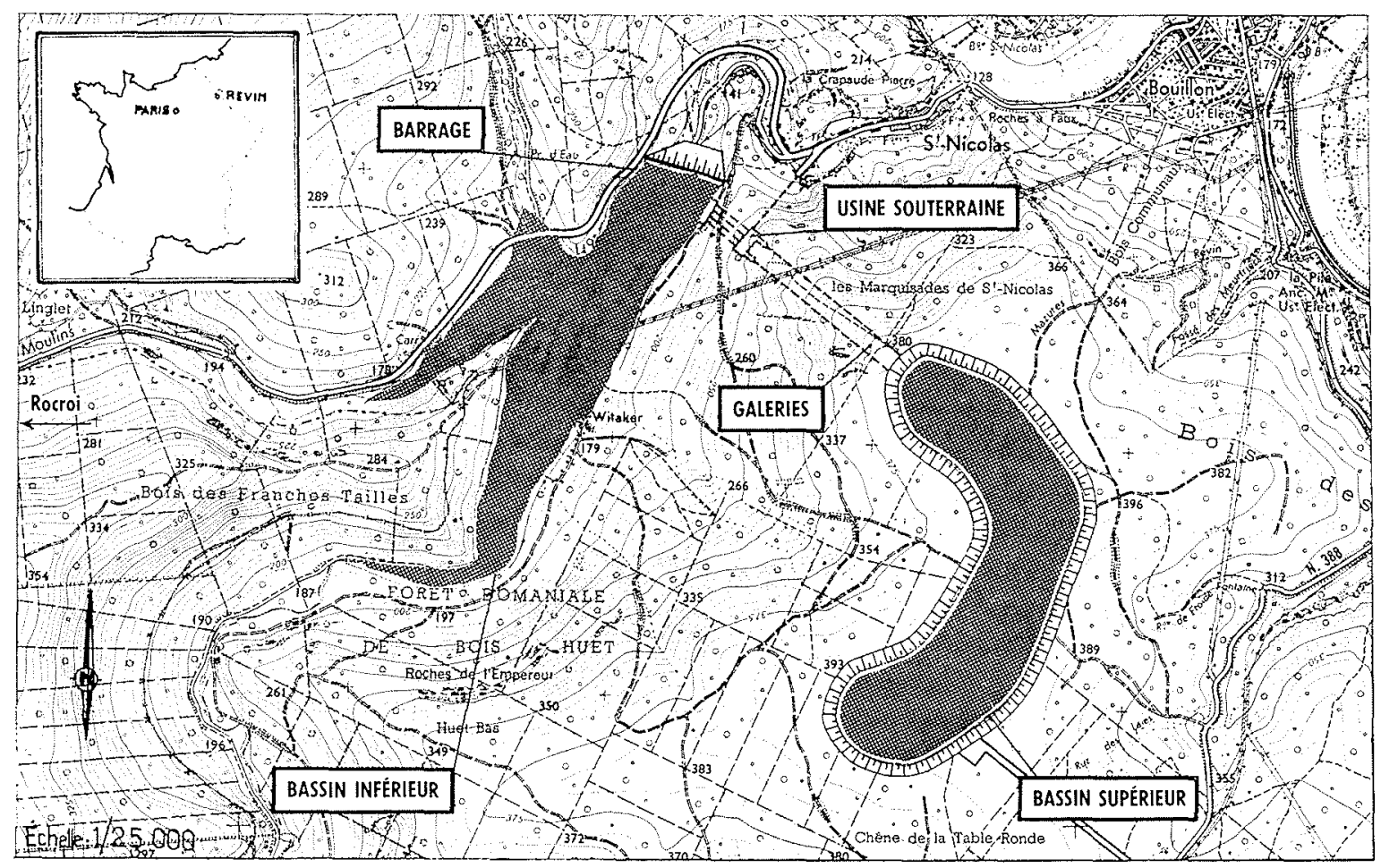

1/ Plan d'ensemble.

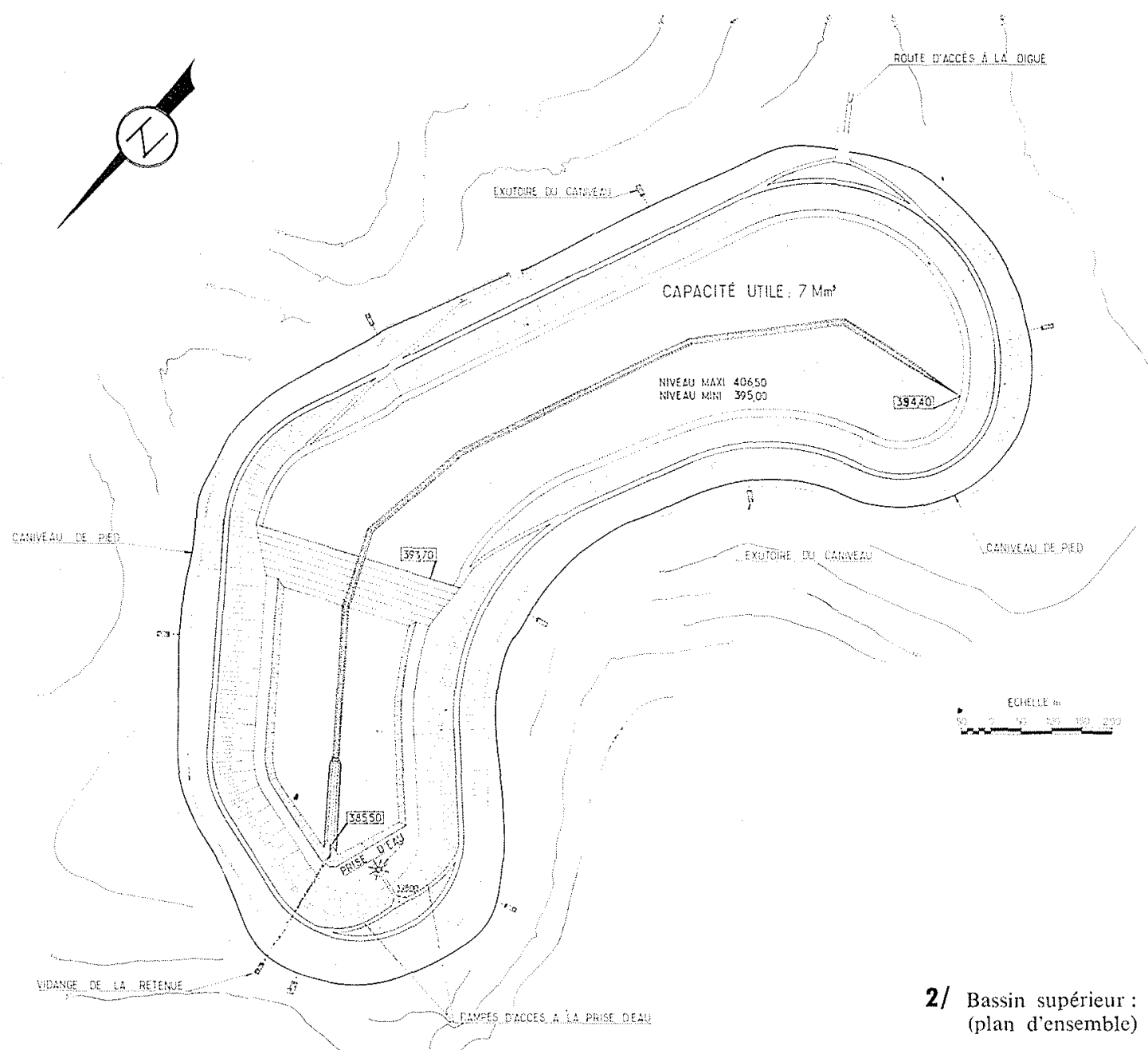



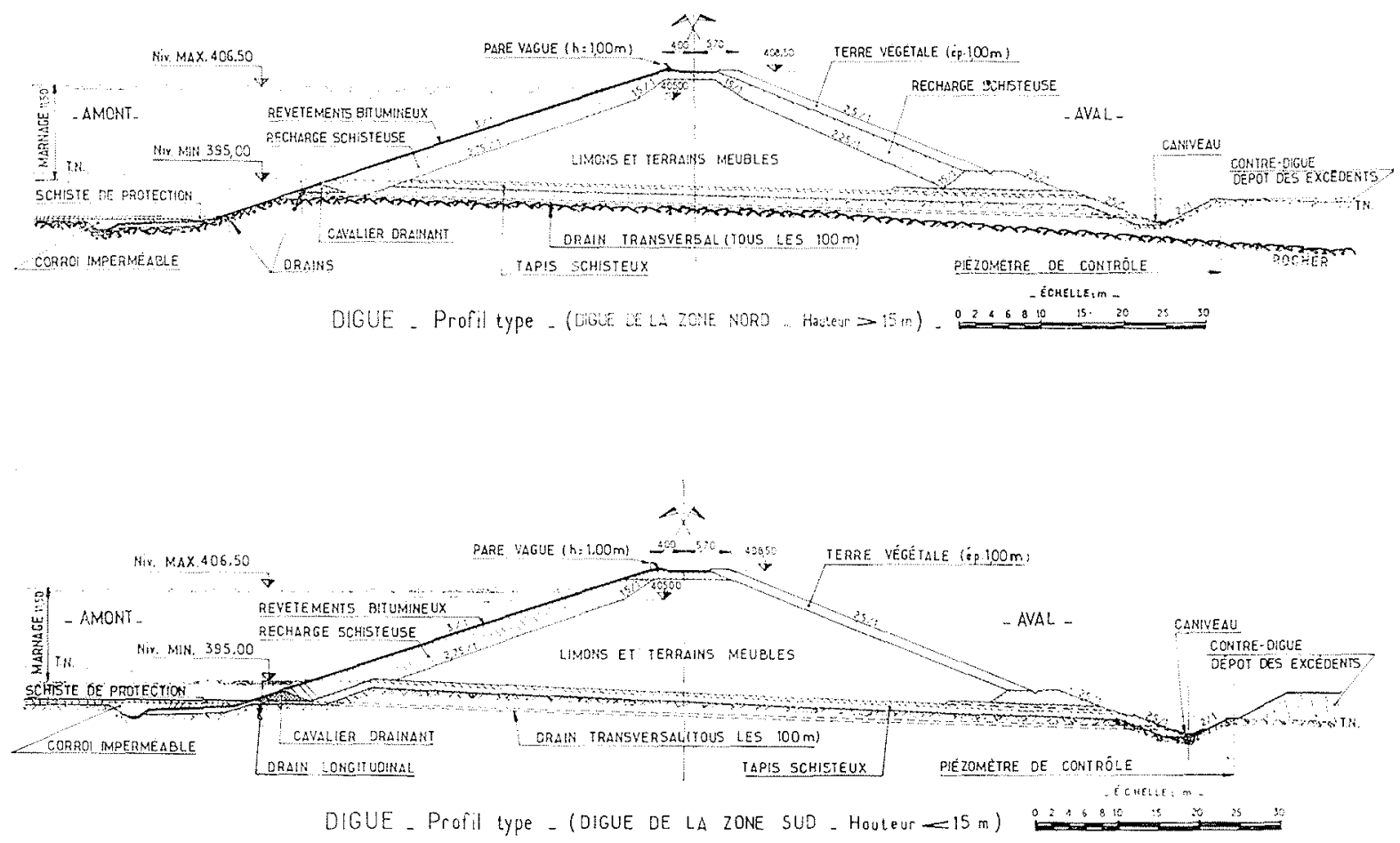

3/

composé de $6 \mathrm{~h}$ de turbinage et $8 \mathrm{~h}$ de pompage, dont le rendement global est de l'ordre de $72 \%$.

Les travaux définitifs ont démarré dans le courant du deuxième semestre 1970; la mise en eau est prévue dans le courant de l'été 1973, la mise en service de la centrale interviendra au cours de l'année 1974.

\section{3. - Description des principaux ouvrages}

\subsection{Le bassin supérieur (fig. 2 et 3 ).}

Le bassin artificiel est réalisé en arasant le sommet du plateau au niveau du fond du bassin et en constituant, avec le déblai ainsi obtenu, une ceinture de digues.

Les digues ont une longueur totale de $4200 \mathrm{~m}$ et une

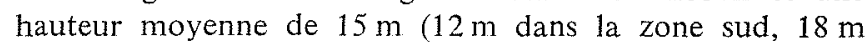
maximum dans la zone nord au voisinage de la prise). Elles représentent un terrassement global de l'ordre de $4 \mathrm{Mm}^{3}$.

Le volume utile, $7 \mathrm{Mm}^{3}$, correspond à une hauteur de marnage de $11,50 \mathrm{~m}$.

- Le fond $d u$ bassin est légèrement incliné vers la prise d'eau. $\mathrm{Au}$ niveau minimal d'exploitation, il reste entièrement couvert d'un matelas d'eau de $0,70 \mathrm{~m}$ d'épaisseur minimale. Il est largement surcreusé dans la zone nord pour procurer les matériaux schisteux utiles pour les recharges des digues. Le reste du bassin est excavé dans des limons et schistes entièrement décomposés en divers produits meubles. Ce fond naturellement presque imperméable est revêtu d'un corroi argileux protégé par une couche de schistes brisés.

- Le parement intérieur des digues est réglé au fruit de $3 / 1$ et revêtu d'un bi-couche de béton bitumineux imper- méable reposant sur un béton bitumineux drainant de forte perméabilité.

- Le parement extérieur des digues est réglé au fruit de $2,5 / 1$ et protégé du gel par une recharge de terre végétale de $1 \mathrm{~m}$ d'épaisseur.

- Le corps des digues est constitué d'un remblai en terrain meuble reposant sur un tapis schisteux relativement drainant. Une recharge schisteuse est interposée à l'amont entre le corps de la digue et le revêtement bitumineux. Une recharge schisteuse aval est prévue dans les zones où la digue est la plus haute.

- Le système de drainage comprend une buse longitudinale de pied amont implantée à la base du bitumineux drainant. Cette buse débouche dans des buses transversales mises en place sous la digue à l'espacement d'une centaine de mètres. Ce réseau de buses est doublé par des recharges drainantes.

- La prise d'eau a la forme d'un cône peu évasé dont le couronnement est fractionné par des piles anti-vortex. Elle n'a ni vannes, ni grilles.

\subsection{Le bassin inférieur (fig. 4).}

Le bassin inférieur est créé par une digue de $36 \mathrm{~m}$ de hauteur et d'environ $300 \mathrm{~m}$ de longueur en crête, dont la construction nécessite un volume de remblais de l'ordre de $700000 \mathrm{~m}^{3}$.

La tranche utile de la retenue correspond à une hauteur de marnage de $16 \mathrm{~m}$.

Le barrage comporte:

- un noyau central imperméable confectionné avec des matériaux meubles empruntés dans un éboulis de pente proche du site; 


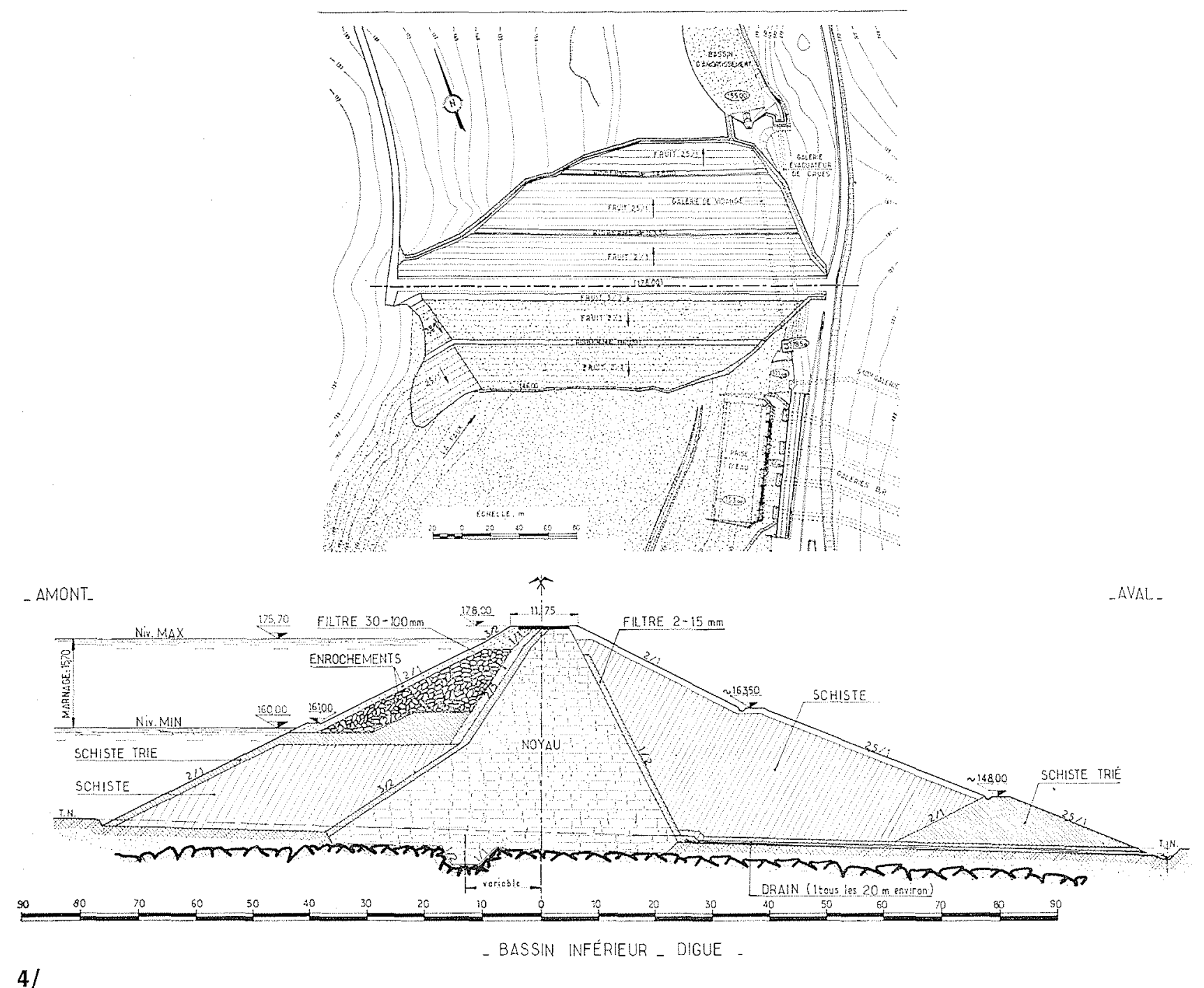

$4 /$

- d'importantes recharges rocheuses amont et aval essentiellement constituées par le mareing provenant des différentes excavations de l'aménagement.

Des enrochements sélectionnés de carrière sont importés pour constituer la partie haute de la recharge amont qui est sollicitée par les marnages rapides.

Des filtres sont interposés entre les recharges et le noyau; un tapis de terre végétale protège le parement aval de la digue.

Les principales sorties d'eau du bassin : adductions, prises d'eau de réfrigération, vidange de fond, évacuateur de crues sont regroupées en un seul ouvrage implanté en rive droite de la retenue: la prise d'eau (fig. 5).

- les adductions débouchent dans la partie basse de l'ouvrage par quatre pertuis doubles, équipés de huit vannes plates $(3 \mathrm{~m} \times 7 \mathrm{~m})$ et protégés par des grilles;

- un mur déversant - aménagé sur le couronnement de la prise d'eau - permet l'évacuation des débits de crues dans un chenal latéral. Une galerie à forte pente conduit les eaux à l'aval du barrage où la dissipation de l'énergie est réalisée par une cuillère. Cet ouvrage est prévu pour l'évacuation de la crue décamillénaire, soit un débit de l'ordre de $150 \mathrm{~m}^{3} / \mathrm{s}$.

L'évacuation des apports naturels de la rivière et le réglage du stock de la retenue sont effectués par l'intermédiaire des ouvrages existants d'un petit aménagement hydro-électrique implanté en rive gauche de la retenue et dont la prise dans le bassin inférieur est réaménagée.

\subsection{Les adductions (fig. 6).}

- Les adductions haute pression comportent un puits vertical de $215 \mathrm{~m}$ de hauteur et un réseau de galeries à faible pente qui relient la base du puits à la centrale sur une longueur d'environ $900 \mathrm{~m}$.

Ces adductions ne sont blindées que sur les 250 derniers mètres à proximité de l'usine.

Le puits a un diamètre de $9 \mathrm{~m}$.

Au niveau du pied de puits, une bifurcation répartit le débit dans deux galeries bétonnées de $7 \mathrm{~m}$ de diamètre et $650 \mathrm{~m}$ de longueur. Chacune de ces galeries est prolongée par une galerie blindée de $5,20 \mathrm{~m}$ de diamètre sur une longueur de $200 \mathrm{~m}$. Ces deux galeries blindées se divisent par deux bifurcations métalliques en quatre rameaux blindés d'une trentaine de mètres de longueur.

- Les adductions basse pression comprennent quatre galeries indépendantes de $6 \mathrm{~m}$ de diamètre et $200 \mathrm{~m}$ de longueur. Chaque adduction comporte une descenderie à forte pente prolongée par une galerie inclinée à $10 \%$ jusqu'au seuil de la prise d'eau. Seule la descenderie est blindée. 


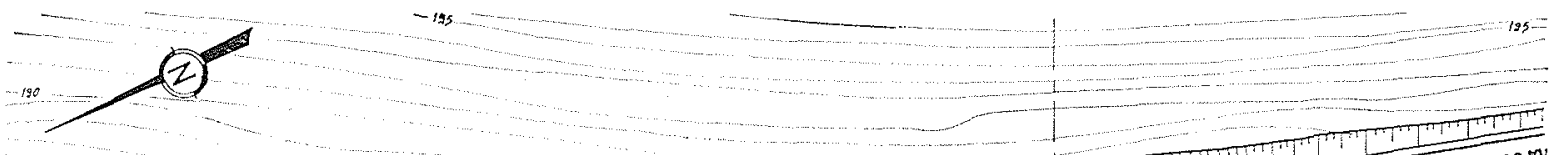

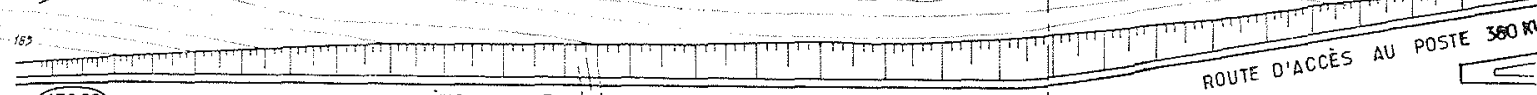

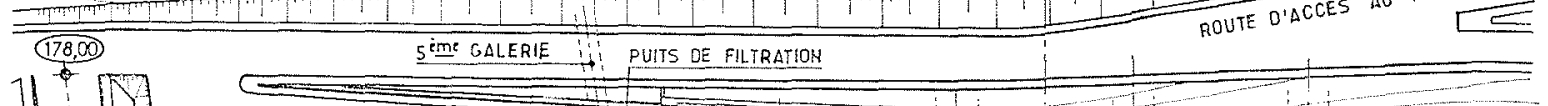

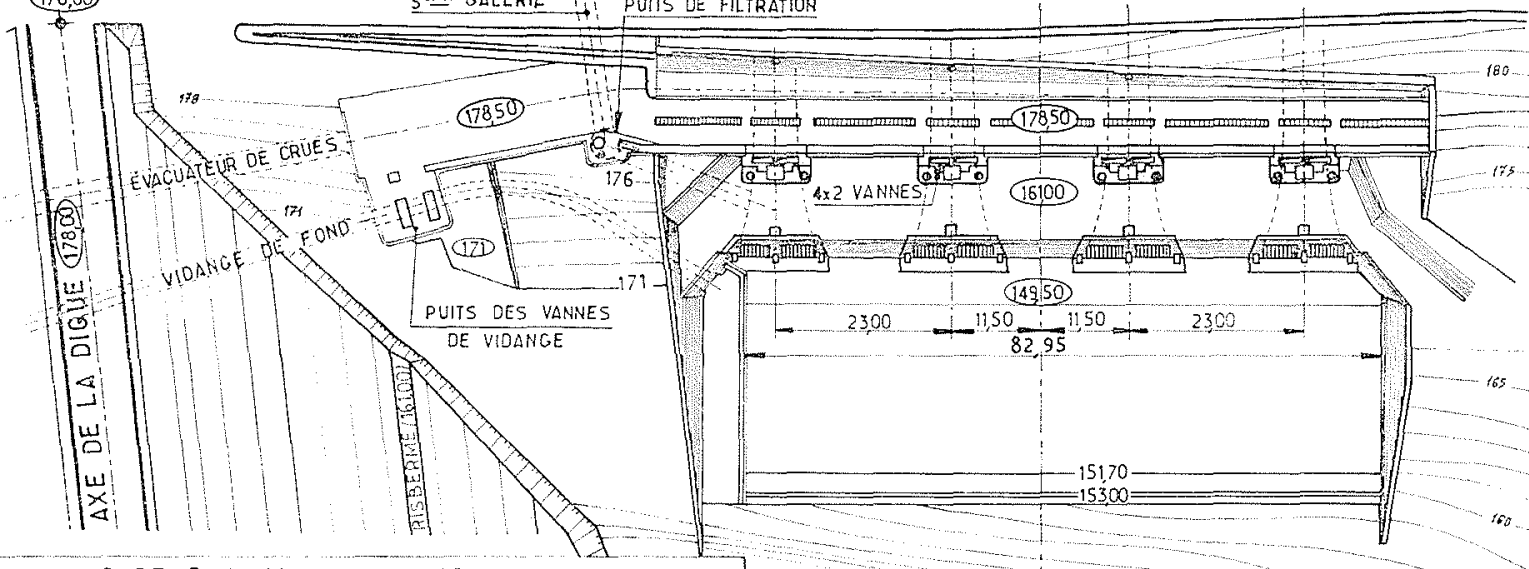

- COUPE DANS L'AXE D'UN GROUPE -

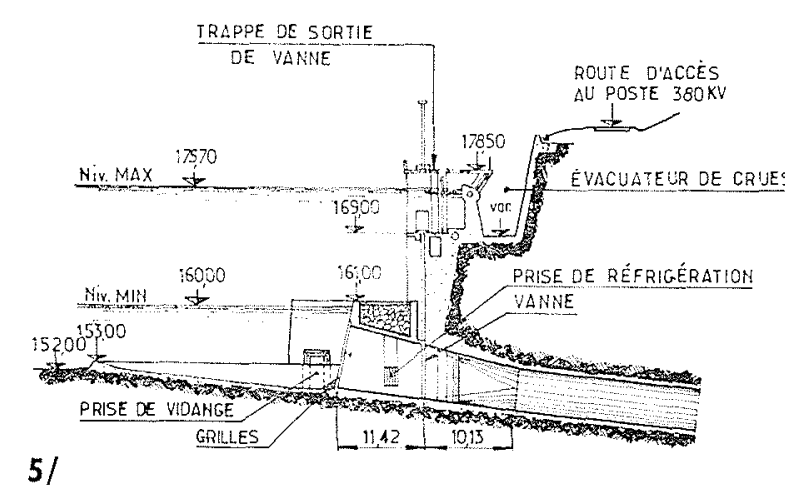

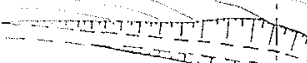

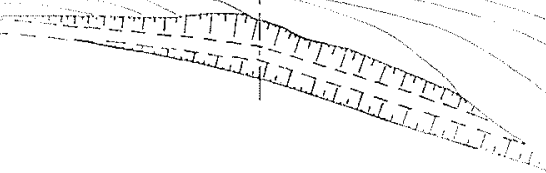

ETELLE: $m$

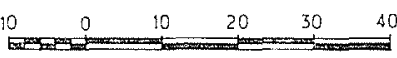

- BASSIN INFÉRIEUR - PRISE dDEAU - Vue en plon.

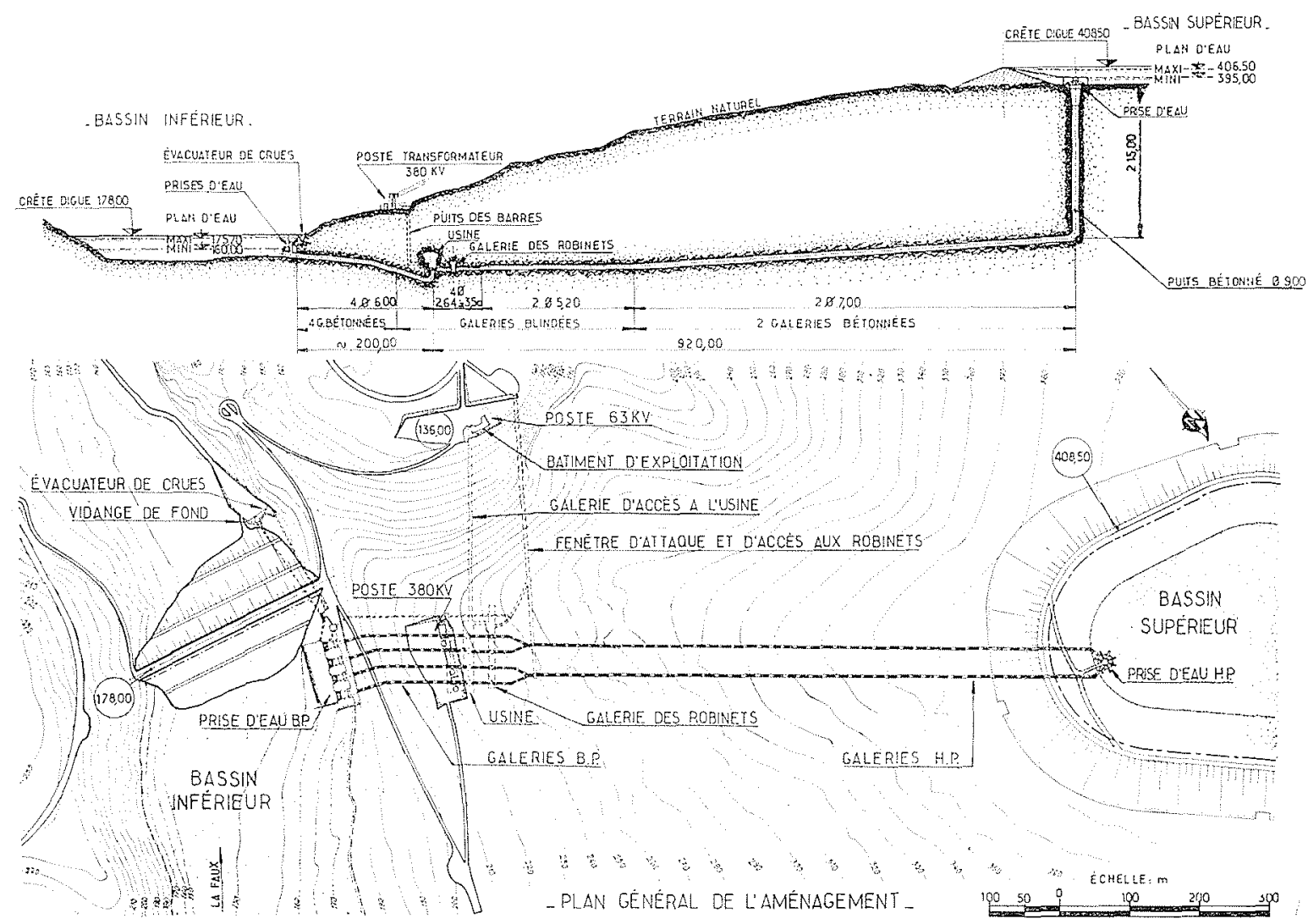

6/ 


\section{J. PONSY}
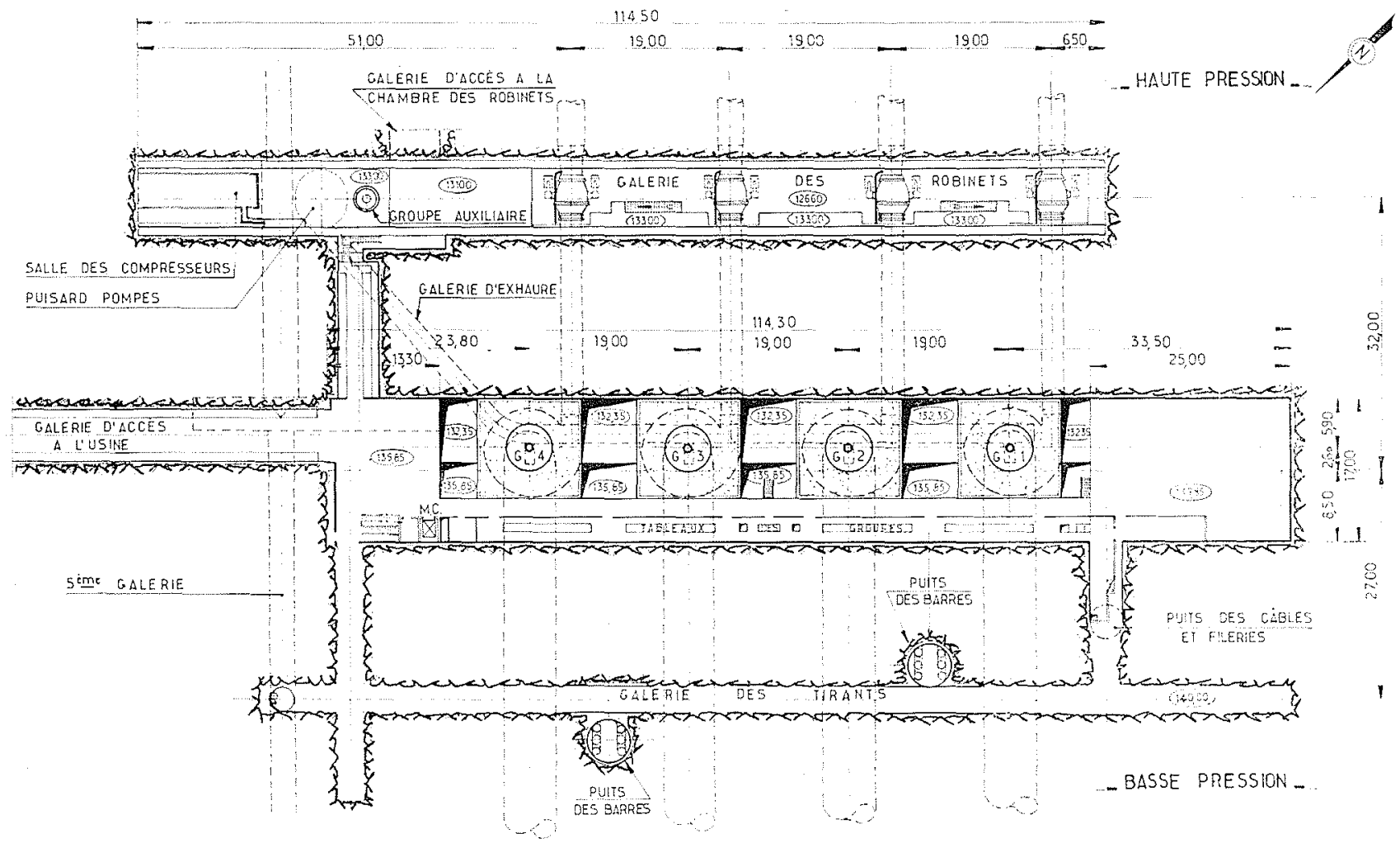

USTE . We en pian -

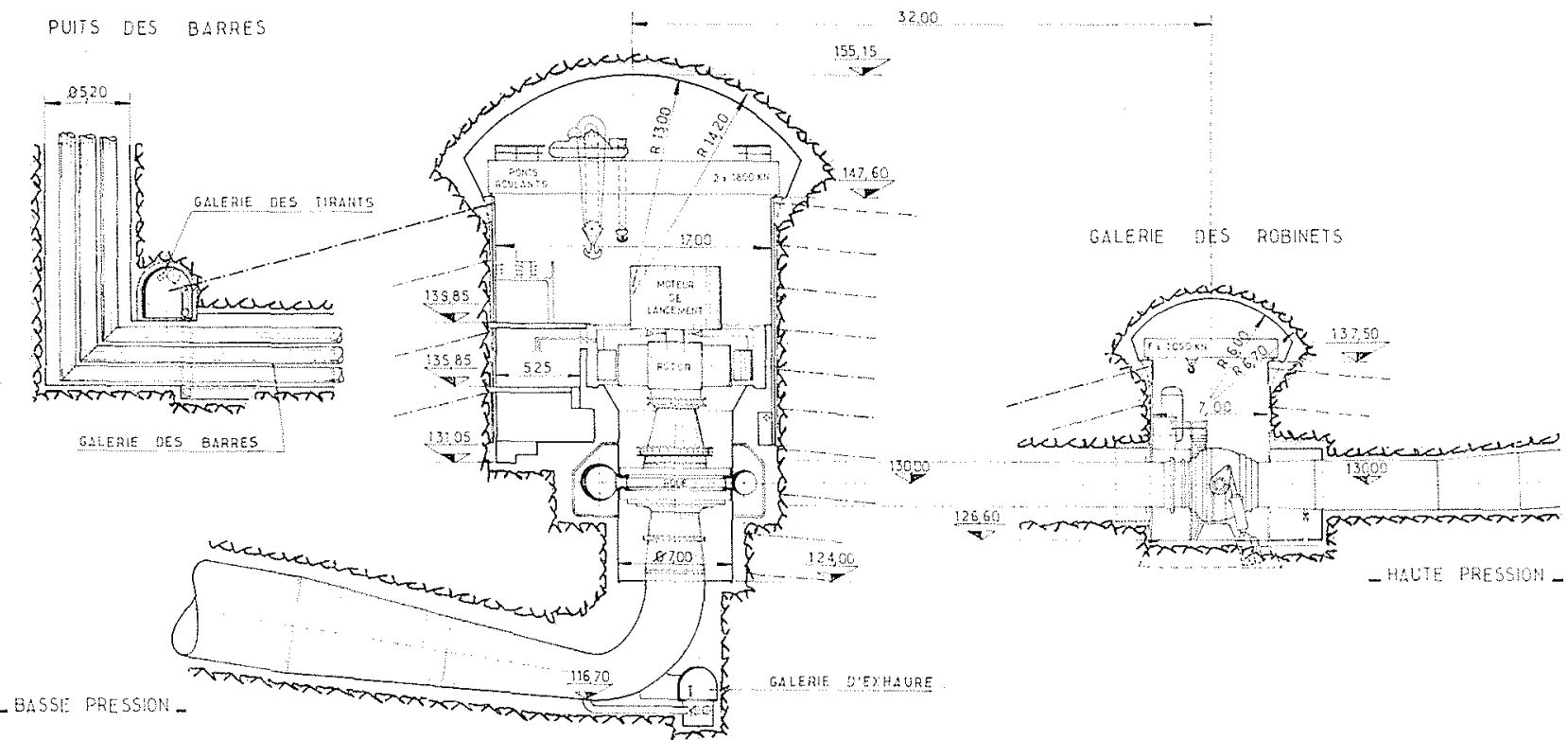

8/

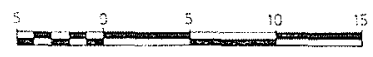




\subsection{Lusine souterraine (fig. 7 et 8).}

L'usine comporte deux cavernes: la salle des machines et la chambre des robinets.

- La salle des machines a une longueur de $114 \mathrm{~m}$ pour une largeur entre piédroits de $17 \mathrm{~m}$. La caverne - qui représente un volume d'excavation de l'ordre de $50000 \mathrm{~m}^{3}-$ a une hauteur maximale entre le fond des aspirateurs et la calotte d'environ $40 \mathrm{~m}$.

La voûte bétonnée supporte par l'intermédiaire de voiles de béton armé les poutres des ponts-roulants. Les parois verticales sont protégées par une gunite.

Deux ponts-roulants de $200 \mathrm{t}$ desservent l'usine qui comporte une plage de montage à chaque extrémité.

L'usine est équipée de quatre groupes Francis réversibles de $180 \mathrm{MW}$; leur vitesse de rotation est de $300 \mathrm{tr} / \mathrm{mn}$ dans les deux sens. Un moteur asynchrone permet le lancement de la machine en pompage, la roue étant dénoyée.

L'énergie est évacuée par deux puits de $80 \mathrm{~m}$ de hauteur jusqu'à quatre transformateurs $(13 / 380 \mathrm{kV}$ de $200 \mathrm{MVA})$ implantés sur une plate-forme extérieure à l'aplomb de l'usine.

- La chambre des robinets de dimensions plus réduites contient les quatre robinets sphériques $(\varnothing 2,64 \mathrm{~m})$, le groupe turbine-pompe auxiliaire et divers matériels auxiliaires.

La disposition des lieux permet l'évacuation gravitaire des eaux jusqu'au niveau du plancher-turbine malgré l'enfoncement de la roue $30 \mathrm{~m}$ en-dessous du niveau minimal d'exploitation du bassin inférieur.

Les accès sont réalisés par deux galeries de $200 \mathrm{~m}$ de longueur.

\section{4. - Examen de quelques problèmes particuliers}

On n'abordera ici que quelques-uns des problèmes qui requièrent l'attention du «génie-civiliste» dans l'étude des projets de stations de pompage.

\subsection{Marnages.}

L'exploitation des retenues de pompage est caractérisée :

- par le nombre et la fréquence très élevés des cycles de vidange et remplissage;

- par la rapidité des variations des plans d'eau; à Revin, par exemple, les plans d'eau descendent à une vitesse de l'ordre de $2 \mathrm{~m} / \mathrm{h}$.

Par suite, ces marnages constituent un facteur particulièrement important, d'une part pour le dimensionnement et le choix des parements amont des digues qui sont directement sollicités par les mouvements d'eau, d'autre part pour la définition des hypothèses de calcul de la stabilité des ouvrages.

\subsubsection{Parements amont des digues de Revin}

Les matériaux disponibles sur le site pour la construction des digues peuvent être regroupés en deux catégories: les matériaux meubles et les matériaux schisteux:

- les matériaux meubles de granulométrie fine, sont très peu perméables et impropres à la confection d'un parement amont de digue car ils sont gélifs et n'ont aucune tenue à l'érosion provoquée par le batillage et les marnages;
- les matériaux schisteux correspondent à un rocher de qualité très moyenne qui subit une fragmentation importante à l'extraction et à la mise en cuvre. Ils comportent une proportion variable mais relativement forte d'éléments fins. L'utilisation de ces matériaux en parement amont ne peut être envisagée car ils n'assureraient pas une protection suffisante contre l'érosion. De plus, leur perméabilité est trop faible pour que l'essorage des recharges suive sans un décalage important la baisse rapide du plan d'eau. Enfin, il est à craindre que les nombreux marnages ne provoquent une migration de «fines» avec les risques de colmatage et de désordres qu'il pourrait en résulter.

En conclusion, les matériaux rocheux de bonne qualité qui conviendraient pour la protection des parements amont n'existent pas sur le site et doivent être importés depuis des carrières de quartzites, distantes de plus de $20 \mathrm{~km}$.

Dans ces conditions, on peut envisager trois solutions pour résoudre le problème de la protection du parement:

a) Un revêtement perméable de béton ou béton bitumineux qui ne joue qu'un rôle de protection mécanique, le corps de digue assurant l'étanchéité. Les risques que présente ce revêtement vis-à-vis du désenrobage par les vases ou du colmatage ne lui confèrent pas les garanties de pérennité souhaitable. De plus, il n'évite pas toutes possibilités de dégradations derrière les revêtements en raison de la rapidité et de la fréquence des marnages. Pour ces raisons, cette solution a été abandonnée.

b) Un revêtement imperméable protégé par un drainage efficace.

c) Un revêtement rocheux, des filtres étant interposés entre cette protection et le corps de digue qui assure l'étanchéité.

Ces deux dernières solutions ont été retenues : la première pour les digues du bassin supérieur, la deuxième pour le barrage du bassin inférieur. Ces choix ont été déterminés par des considérations essentiellement économiques.

\section{Bassin supérieur.}

Un revêtement rocheux du parement eut nécessité l'importation de $400000 \mathrm{~m}^{3}$ de matériaux - enrochements et filtres - provenant de carrières éloignées. En raison du transport et des sujétions liées à l'insuffisance du réseau routier, le coût de cette protection eut été notablement plus élevé que celui de la solution retenue :

La protection amont est donc réalisée par un revêtement imperméable de béton bitumineux.

Cette solution présente plusieurs avantages : tout d'abord, elle est la plus économique parce qu'elle correspond au volume minimal de matériaux importés $\left(60000 \mathrm{~m}^{3}\right)$; par ailleurs, le risque de migration d'éléments fins dans la recharge schisteuse est pratiquement éliminé; enfin, l'exécution des terrassements est simplifiée parce qu'elle ne comporte pas la mise en place, toujours délicate, de filtres sur de grandes surfaces, parce que d'autre part elle permet de réaliser un remblai meuble sans zonage, un peu moins soigné et donc plus aisé; en effet, dans ce projet, l'homogénéité et l'imperméabilité des remblais meubles ne sont plus aussi essentielles que dans l'autre solution où ces remblais constituent un véritable noyau d'étanchéité. Ce dernier point était très important à Revin car, d'une part les matériaux meubles du site sont assez médiocres, hétérogènes et sensibles à l'eau, d'autre part, les conditions climatologiques sont peu favorables à la réalisation de terrassements. 


\section{J. PONSY}

Ce revêtement comporte aussi ses inconvénients : on doit, en effet, envisager l'éventualité d'une défaillance de l'étanchéité du revêtement qui provoquerait une saturation de la recharge schisteuse. Ce risque conduit à adopter le même fruit pour le parement amont que dans la solution « protection rocheuse $\gg$ et impose de prévoir un réseau de drainage efficace et sûr.

\section{Bassin inférieur.}

La protection amont est réalisée par une recharge rocheuse constituée :

- par des enrochements de carrière dans la zone du marnage soit sur une hauteur de l'ordre de $18 \mathrm{~m}$;

- dans la partie basse de l'ouvrage par des matériaux schisteux dont les caractéristiques sont ici suffisantes car cette zone n'est sollicitée que par les vidanges exceptionnelles qui s'effectuent lentement.

Dans ces conditions, la comparaison économique - protection rocheuse, revêtement étanche - est en faveur de la solution rocheuse mais l'écart est peu significatif et, en fait, le choix a été fixé par des considérations de sécurité et d'entretien :

En raison de la hauteur de l'ouvrage $(40 \mathrm{~m})$ et de la qualité drainante imparfaite des recharges schisteuses, une digue à masque amont aurait présenté dans le cas d'une rupture locale du masque des conditions de stabilité à la vidange rapide très délicates pour le talus amont. Par ailleurs, le revêtement rocheux présente l'avantage de limiter les travaux à un chantier de terrassement pur et de constituer une solution rustique qui comporte peu de sujétions d'entretien.

Les inconvénients de cette solution sont limités par comparaison aux avantages précités: les sujétions de mise en place des filtres n'intéressent qu'une surface réduite; d'autre part, le volume supplémentaire de matériaux importés de carrière est raisonnable $\left(50000 \mathrm{~m}^{3}\right)$ et le transport peut s'effectuer par le réseau routier existant.

\subsubsection{Hypothèses de STABILITÉ DES DIGUES.}

La rapidité de descente du plan d'eau conduit à retenir des hypothèses très sévères pour l'étude de la stabilité des digues pendant les vidanges.

\section{Bassin supérieur.}

Dans la zone Sud, les digues sont fondées au terrain meuble très peu perméable qui surmonte de plusieurs mètres un niveau relativement plus perméable correspondant au niveau de schistes brisés du toit du bed-rock (fig. 9).

Un drainage s'établira de façon diffuse depuis le fond du bassin jusqu'à l'extérieur des digues à travers des terrains dont la capacité drainante est très faible. L'étude de l'évolution de cet écoulement au cours d'une vidange rapide conduit à penser que la pression dans la fondation est plus longue à se «détendre » que le lac à se vider, à cause de l'air occlus comprimé et de la faible perméabilité des terrains. Le niveau des schistes brisés constitue alors un réservoir d'eau sous pression qui crée un gradient de pression dirigé vers le haut. On a donc retenu l'hypothèse que la pression sous le manteau de terrains meubles restait la même qu'avant vidange, sa valeur étant toutefois limitée à un maximum équivalent au poids de terre du niveau correspondant.

Dans la zone nord, les digues sont pratiquement fondées au niveau rocheux plus perméable à travers lequel s'effectuera le drainage jusqu'au caniveau extérieur. En cas de vidange rapide, il n'y a pas lieu de retenir l'hypothèse pré- cédente: compte tenu de la faible déformabilité et de la relative perméabilité des matériaux de fondation, la dissipation des pressions sera suffisamment rapide pour que n'existe pas le même risque de sous-pressions.

\section{Bassin inférieur.}

On ne peut admettre que le régime hydraulique à l'intérieur du noyau est en équilibre à tout instant avec les conditions aux limites nouvelles imposées par la vidange, en raison de la rapidité de cette opération, des caractéristiques de perméabilité et déformabilité du matériau et de la présence d'air occlus dans le terrain ou dissous dans l'eau. En conséquence, il a été retenu l'hypothèse que les pressions interstitielles du noyau restaient figées pendant les vidanges à leur valeur initiale.

\subsection{Adductions. - Blindages.}

\subsubsection{IMPLANTATION SOUTERRAINE.}

Les adductions des stations de pompage sont en totalité ou en grande partie souterraines en raison de l'important enfoncement de l'usine; il faut en effet caler les roues des machines une trentaine de mètres en-dessous du niveau minimal d'exploitation du bassin inférieur pour limiter les risques de cavitation pendant le fonctionnement en pompe.

A Revin, les adductions sont entièrement souterraines car, en raison de la topographie assez molle du plateau, une conduite extérieure ne plongerait pas assez vite dans la partie haute pour être à l'abri des coups de bélier de dépression.

\subsubsection{BlindAGES. - IMPERMÉABILITÉ DU ROCHER.}

L'importance des débits d'équipement conduit à la réalisation de galeries de grande section qui sont soumises à des pressions de service relativement élevées; les blindages métalliques qui assurent l'étanchéité de ces ouvrages représentent un poste très lourd du devis. L'implantation de ces adductions dans une roche imperméable peut permettre la suppression - tout au moins partielle — des blindages; on conçoit alors l'importance pour ce type d'ouvrage des caractéristiques de perméabilité du rocher, car ce facteur intervient non seulement sur l'économie du projet mais aussi sur le choix du schéma d'adductions et de la position de l'usine sur ces adductions :

- dans un terrain perméable, les études de dimensionnement économique conduiront à raccourcir au maximum les adductions haute pression et à implanter l'usine sous le bassin supérieur; à l'inverse, dans une roche imperméable, on aura tendance à allonger les adductions haute pression, ce qui permettra de reporter l'usine près du bassin inférieur et de supprimer la cheminée d'équilibre sur les adductions basse pression;

- dans un terrain imperméable, les sections des galeries ne seront limitées que par les caractéristiques mécaniques de la roche; dans un terrain perméable, l'épaisseur maximale des blindages introduira une nouvelle limite qui pourra imposer un fractionnement plus important du réseau d'adduction haute pression.

\subsubsection{ADDUCTION DE REVIN.}

L'imperméabilité du massif a été testée par un essai en caverne, de nombreux indices très favorables ayant été préalablement recueillis par des essais d'eau en sondages (fig. 10)

A l'extrémité d'une galerie de reconnaissance de $3 \mathrm{~m}$ de diamètre, deux chambres successives d'une trentaine de 


\section{I/REGIME PERMANENT}

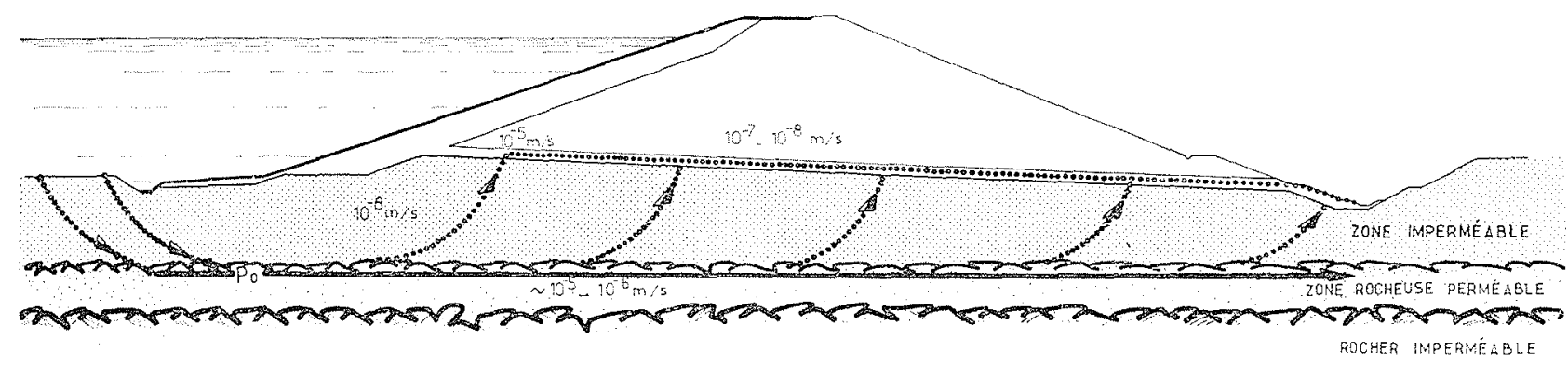

I/ APRES VIDANGE RAPIDE

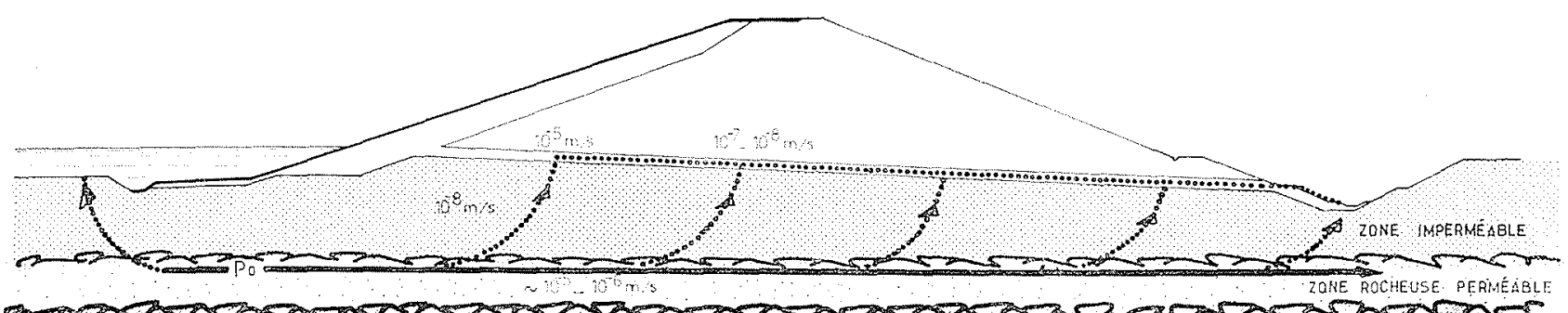

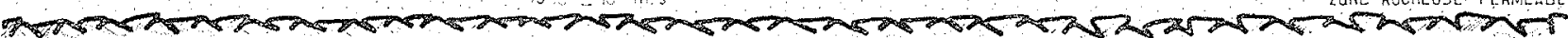

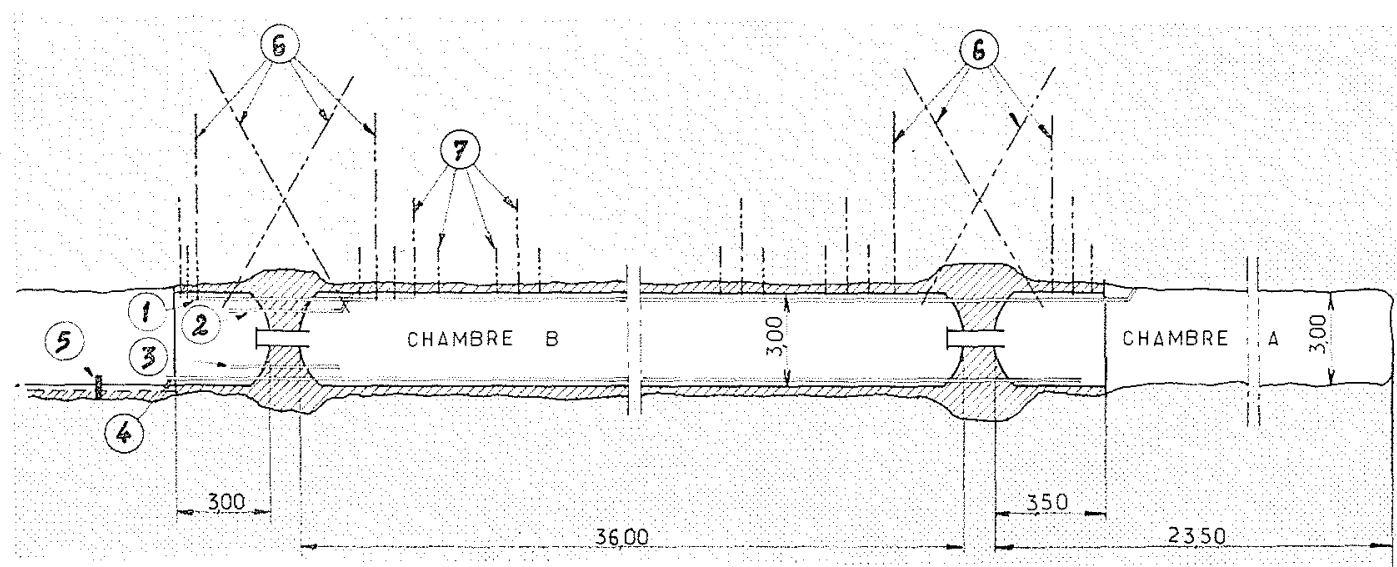

(1) PURCE ChaMbRe $\triangle$

(2) Purge chambre $B$

(3) Vidange. remplissage chambre 8

(4) vidange. remplissage chambre a

É CHELLE On $m$

(5) Deversoir de Mesure des fuites

(6) INJECTION BOUCHONS

(7) INJECTION CHAMBRE $B$

- ADDUCTIONS _ esSAIS dE PERMÉABILITÉ eN CAVERNE -

$10 /$ 


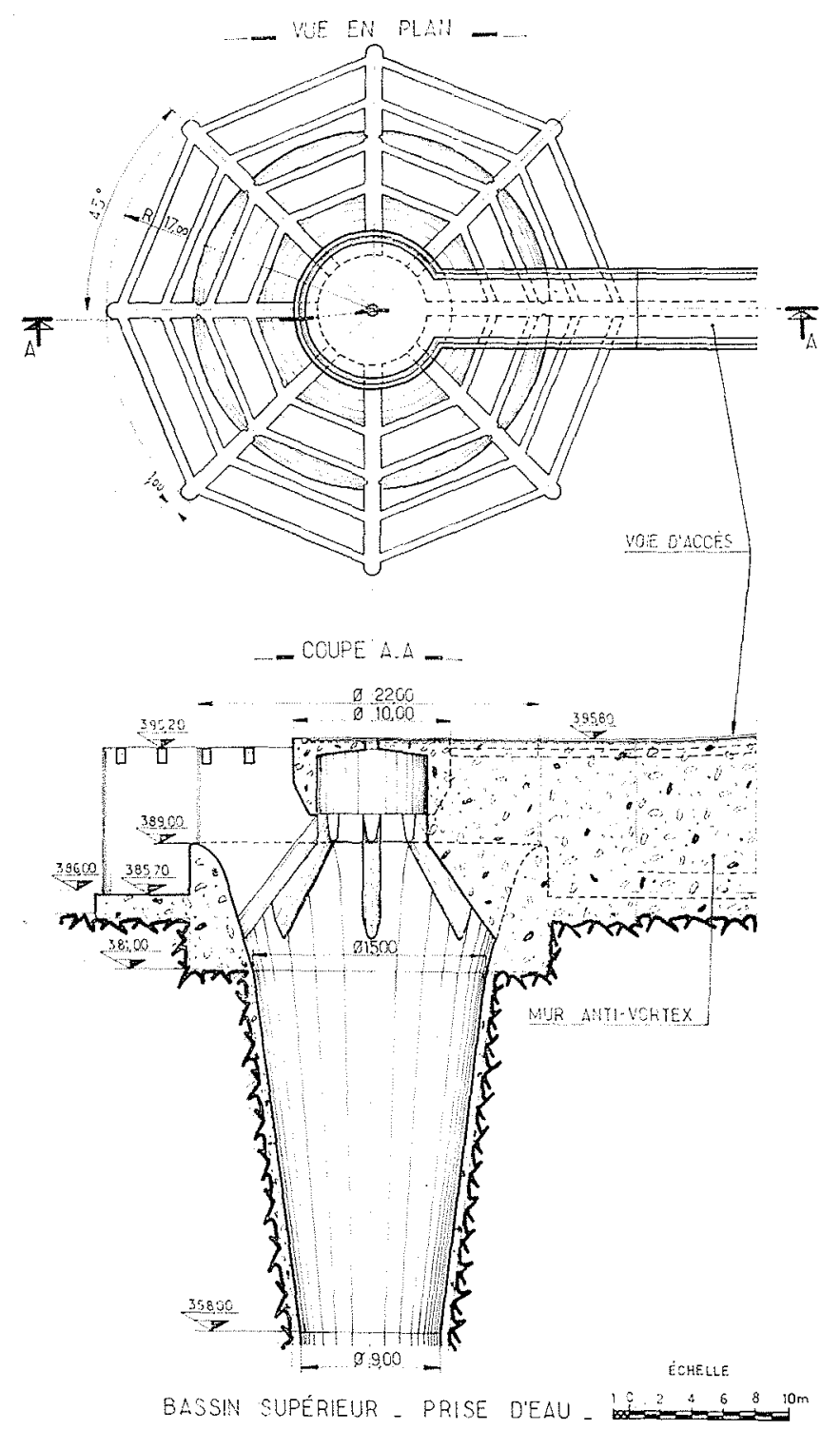

$11 /$

mètres de longueur ont été isolées par des bouchons de béton; la première était brute d'excavation, la deuxième revêtue. Ces deux chambres, implantées sous une couverture de l'ordre de $180 \mathrm{~m}$, ont été portées séparément et par paliers successifs à des pressions de 40 bars; le dispositif installé permettait de mesurer simultanément la pression, le débit injecté et le débit de fuite en galerie. Les fuites sont restées négligeables (inférieures à $0,11 / \mathrm{mn} / \mathrm{ml}$ galerie).

Cette remarquable étanchéité du massif permet la suppression des blindages sur la plus grande partie de l'adduction haute pression :

- les blindages ne sont conservés que sur une longueur de $250 \mathrm{~m}$ dans le tiers aval où la couverture rocheuse est insuffisante; dans cette zone, en effet, n'est plus respectée la condition que la couverture de terrain doit être au moins égale à la moitié de la charge d'eau - critère classique et relativement prudent compte tenu de la forte densité du rocher $\left(2,8 \mathrm{t} / \mathrm{m}^{3}\right)$;

- à l'amont, le puits et les galeries sont entièrement revêtus. Ce revêtement est nécessaire pour améliorer le coefficient d'écoulement et donc réduire les pertes de charge. De plus, il constitue une sécurité vis-à-vis de la stabilité de l'excavation et des fuites locales imprévisibles, car il permet - par des injections sous forte pression - de reconstituer, tout au moins partiellement, les caractéristiques de l'anneau de rocher décomprimé par le tir. Ces revêtements ne sont pas armés car la fissuration des bétons par les pressions internes élevées (25 à 35 bars) devrait rester, compte tenu du blocage au rocher - dans des limites compatibles avec la sécurité de l'ouvrage.

\subsection{Prises d'eau.}

Ces ouvrages sont à la fois des ouvrages de prise et de rejet d'eau dans les bassins. Par suite, les variations de section qu'ils présentent créent des écoulements convergents ou divergents suivant le type de fonctionnement, pompage ou turbinage, et bien évidemment ce sont les conditions de divergence qui imposent le choix des formes hydrauliques.

C'est ainsi que pour la prise d'eau du bassin supérieur (fig. 11), le fonctionnement en pompe impose la définition du cône de raccordement; pour que les pertes de charge restent dans des limites acceptables, on est conduit à allonger ce cône et à réduire son angle d'ouverture, plus qu'il ne serait nécessaire pour obtenir de bonnes conditions hydrauliques d'entonnement en turbinage.

Par contre, pour que cet entonnement s'effectue dans des conditions satisfaisantes on doit prévoir un dispositif antitourbillonnaire : il est constitué par des pilettes qui fractionnent le seuil en huit pertuis et portent sur leur couronnement des poutres transversales.

Les études sur modèle réduit ont conduit à compléter ce dispositif par un mur, reliant la prise à la digue, qui s'est révélé le moyen le plus efficace pour s'opposer à la rotation de l'eau au large.

Discussion

Président: M. H. dE Maublanc

M. le Président remercie M. Ponsy pour son exposé qui met bien en relief les sujétions particulières résultant du fonctionnement de ce type d'installation pour certains ouvrages de Génie Civil, tels que canaux d'amenée et de fuite, cheminée d'équilibre, etc.

Il indique que les travaux de la station de Revin sont actuellement en cours; les terrassements du réservoir supérieur sont terminés et on procède aux revêtements; on poursuit l'exécution des adductions souterraines ainsi que l'aménagement du bassin aval. La mise en service de cette puissante installation d'accumulation débutera par la mise en route d'un premier groupe au cours de l'hiver 19731974 ;

Le démarrage des autres groupes s'échelonnera sur l'année 1974.

La discussion s'ouvre par la question ci-après, posée par M. LÉo : Le problème de la turbidité de l'eau se pose-t-il à Revin? Des dispositifs particuliers ont-ils été prévus pour éviter le mouvement aller et retour d'une eau chargée de limons?

M. Ponsy répond que le risque de présence d'eaux chargées de limons dans les bassins de Revin paraît très limité car la rivière su laquelle est implanté le bassin inférieur est de faible importance: le bassin versant de la Faux est de $100 \mathrm{~km}^{2}$ environ; le débit moyen annuel ne dépasse guère $1 \mathrm{~m}^{3} / \mathrm{s}$ et les crues annuelles sont de l'ordre de $10 \mathrm{~m}^{3} / \mathrm{s}$

De plus, les deux bassins comportent une tranche morte où pour. ront être stockés les dépôts de limons éventuels.

En l'absence d'autres questions, M. le Président clôt la discussion et donne la parole à M. E. MORENon pour l'exposé de sa communication. 